\title{
Performance of careHPV for detecting high-grade cervical intraepithelial neoplasia among women living with HIV-1 in Burkina Faso and South Africa: HARP study
}

Michel Segondy ${ }^{*}$, , Helen Kelly ${ }^{2}$, Mahlape P Magooa ${ }^{3}$, Florencia Djigma ${ }^{4}$, Jean Ngou ${ }^{1}$, Clare Gilham ${ }^{2}$, Tanvier Omar $^{5}$, Olga Goumbri-Lompo ${ }^{6}$, Pamela Michelow ${ }^{5}$, Sylviane Doutre ${ }^{1}$, Omar Clavero ${ }^{7}$, Admire Chikandiwa ${ }^{8}$, Bernard Sawadogo ${ }^{6}$, Marie-Noelle Didelot ${ }^{1}$, Valérie Costes $^{1}$, Nicolas Méda ${ }^{6}$, Sinead Delany-Moretlwe ${ }^{8}$ and Philippe Mayaud ${ }^{2,8}$ for the HARP Study Group

${ }^{1}$ Department of Biology and Pathology, INSERM U1058 and University Hospital (CHU), Montpellier 34000, France; ${ }^{2}$ Department of Clinical Research, London School of Hygiene and Tropical Medicine, London WC1E 7HT, UK; ${ }^{3}$ Centre for HIV and STIs, National Institute for Communicable Diseases, National Health Laboratory Service, Johannesburg 2131, South Africa; ${ }^{4}$ Centre de Recherche Biomoléculaire Pietro Annigoni, Ouagadougou BP7021, Burkina Faso; ${ }^{5}$ Department of Anatomical Pathology, University of the Witwatersrand, and National Health Laboratory Service, Johannesburg 2131, South Africa; ${ }^{6}$ Centre de Recherche Internationale en Santé, University of Ouagadougou, Ouagadougou BP7021, Burkina Faso; ${ }^{7}$ Infections and Cancer Unit, Catalan Institute of Oncology, Barcelona 08908, Spain and ${ }^{8}$ Reproductive Health \& HIV Institute, University of the Witwatersrand, Johannesburg 2001, South Africa

Background: The careHPV assay is a test for high-risk (HR) human papillomaviruses (HPV) detection designed to be affordable in resource-poor settings. We evaluated the performance of careHPV screening among 1052 women living with HIV/AIDS included in the HARP (HPV in Africa Research Partnership) study in Burkina Faso (BF) and South Africa (SA).

Methods: Cervical samples were tested for HR-HPV by the careHPV and the INNO-LiPA HPV genotyping Extra assays. All women had Pap smear testing, visual inspection with acetic acid/Lugol's iodine (VIANILI) and colposcopy. Cervical biopsies were obtained for participants who were HR-HPV DNA positive by careHPV or who had abnormalities detected on cytology, VIAVILI or colposcopy.

Results: Overall, $45.1 \%$ of women had a positive careHPV test (46.5\% in BF, $43.8 \%$ in SA). The careHPV positivity rate increased with the grade of cytological lesions. Sensitivity and specificity of careHPV for the diagnosis of CIN2 $+(n=60$, both countries combined) were 93.3\% (95\% confidence interval (Cl): 83.8-98.2) and 57.9\% (95\% Cl: 54.5-61.2), respectively. Specificity increased with CD4 count. careHPV had a similar clinical sensitivity but higher specificity than the INNO-LiPA assay for detection of CIN2 + .

Conclusions: Our results suggest that careHPV testing is a reliable tool for cervical cancer screening in HIV-1-infected women in sub-Saharan Africa.

Cervical cancer, which is the fourth most common cancer in women worldwide (Ferlay et al, 2015), is the most frequent cancer and the leading cause of cancer death in Sub-Saharan African women (Denny and Anorlu, 2012). Infection with human immunodeficiency virus type $1(\mathrm{HIV}-1)$ is an additional risk factor for the development of precancerous and cancerous cervical

*Correspondence: Dr M Segondy; E-mail: m-segondy@chu-montpellier.fr

Received 3 December 2015; revised 12 May 2016; accepted 12 June 2016; published online 19 July 2016

(c) 2016 Cancer Research UK. All rights reserved 0007 - 0920/16 
lesions (De Vuyst et al, 2008). Therefore, there is a need for developing preventive measures in this highly exposed population of African women living with HIV/AIDS (WLHA). Unfortunately, cervical cancer screening programs are lacking in most Sub-Saharan African countries and the diagnosis of cervical cancer is generally made at an advanced stage of the disease when treatment is unavailable or ineffective.

The development of precancerous cervical lesions that may evolve to invasive carcinoma is associated with persistent cervical infection with carcinogenic types of human papillomaviruses (HPV) designated as high-risk (HR) HPV. It has been shown that detection of HR-HPV in cervical samples is a highly sensitive tool for identifying women at risk of precancerous or cancerous cervical lesions (Cuzick et al, 2008; Ronco et al, 2014), but evaluation among WLHA have only rarely been conducted.

The careHPV assay (Qiagen Corporation, Gaithersburg, MD, USA) is a qualitative test for HR-HPV detection targeting $14 \mathrm{HR}$ HPV types: HPV16, HPV18, HPV31, HPV33, HPV35, HPV39, HPV45, HPV51, HPV52, HPV56, HPV58, HPV59, HPV66 and HPV68. This microplate assay, based on the hybridisation of HRHPV DNA with a cocktail of RNA probes and chemiluminescence signal amplification, was adapted from the Hybrid Capture 2 (HC2) assay (Qiagen) and designed to be simpler, more rapid to use, and more affordable than HC2 in resource-poor settings (Qiao et al, 2008; Gage et al, 2012).

HARP (HPV in Africa Research Partnership) is a research programme conducted among WLHA in Burkina Faso (BF) and South Africa (SA) with the aim to prospectively evaluate several screening approaches for prevention of cervical cancer. In the first round of screening, we have shown that, compared with the INNO-LiPA HPV genotyping Extra assay, HC2 performed well in this population, with a similar sensitivity and a higher specificity for the diagnosis of high-grade cervical intraepithelial neoplasia $(\mathrm{CIN})$ grade 2 or more severe $(\mathrm{CIN} 2+)$. We have already reported an excellent agreement between HC2 and careHPV in a subgroup of HARP participants (Ngou et al, 2013), comparable to what was found in HIV-seronegative women in China (Chen et al, 2014; Lin et al, 2014). However, it remained to be demonstrated that care HPV had a good performance among African WLHA. For this reason, we used the second round of screening in HARP 18 months after enrolment to evaluate the performance of careHPV for the detection of CIN2 + .

\section{MATERIALS AND METHODS}

Study population. Participants were confirmed HIV-1 - seropositive women aged 25-50 years recruited from the HIV outpatient clinic of the University Hospital of Ouagadougou, BF, and HIV treatment centres and surrounding primary health care clinics in Hillbrow, Johannesburg, SA. Eligible women were invited for inclusion in the study if they were resident in the recruitment city, did not had a total hysterectomy or history of cervical cancer treatment and were not pregnant or less than 8 weeks post partum.

Ethical approval was granted from Ministry of Health in Burkina Faso (no. 2012-12-089), the Witwatersrand University in South Africa (no. 110707) and the London School of Hygiene and Tropical Medicine (no. 7400). All women provided a written informed consent at the screening visit, and they were given a reflection period of at least 7 days before enrolment in the study. A second written informed consent was obtained at the enrolment visit for enrolment and follow-up over scheduled visits at months (M) 6, 12 and 18 .

care HPV assay. At the M18 visit, endo- and ectocervical sampling was performed using the careHPV sample collection device consisting of a careBrush and a vial containing $1 \mathrm{ml}$ of careHPV collection medium (Qiagen). After collection, the brush was stirred into the collection medium, the cell collection was homogenised by vortexing and divided into four $0.25-\mathrm{ml}$ aliquots. One aliquot was maintained at $4{ }^{\circ} \mathrm{C}$ until care HPV analysis, performed within less than 4 weeks, and the others were cryopreserved at $-80^{\circ} \mathrm{C}$. The care HPV test was performed using $50 \mu \mathrm{l}$ of cervical sample in collection medium according to the manufacturer's instruction. The tests were performed at the respective sites by medical scientists specifically trained by a Qiagen's scientist. The positive or negative result of the careHPV assay was displayed by the careHPV test controller without additional specification of the luminescent signal intensity.

HPV detection and genotyping. All cervical specimens were tested using the INNO-LiPA HPV genotyping Extra assay (Fujirebio, Les Ulis, France) as previously described (Ngou et al, 2015). This assay, which is based on PCR amplification of HPV DNA using broad-spectrum SPF10 consensus primers followed by hybridisation of the amplicons with type-specific oligonucleotides probes immobilised on membrane strips, allows identification of $28 \mathrm{HPV}$ types, including the $14 \mathrm{HR}-\mathrm{HPV}$ types targeted by the careHPV assay, the possible carcinogenic types HPV26, HPV53, HPV69, HPV70, HPV73 and HPV82, and the low-risk types HPV6, HPV11, HPV40, HPV43, HPV44, HPV54, HPV71 and HPV74. Testing was performed on an aliquot preserved at $-80^{\circ} \mathrm{C}$. A sample was considered HPV + if at least one of the type-specific probes or one of the HPV control probes were detected.

Cytological and histological analysis. An additional cervical brush was collected from the ecto- and endocervix and rolled on a glass slide which was fixed with ethanol for cytological reading using the Papanicolaou method (Pap test). Conventional cytology was used as liquid-based cytology was not available at that time in the African laboratories involved in the study. All participants had visual inspection with acetic acid/Lugol's iodine (VIA/VILI) performed by trained nurses and colposcopy performed by trained colposcopists. Systematic four-quadrant biopsy and directed biopsy from any suspicious lesions were performed for participants testing positive by careHPV or who had abnormalities detected on cytology ( $\geqslant$ ASC-US), VIA/VILI or colposcopy.

The Bethesda system for reporting cervical cytology (Smith, 2002) was used for cytology results and the CIN classification for histology results. Cytological and histological slides were independently examined at each site by two senior pathologists blinded to the other study results. Pathologists were trained before the start of the study in order to harmonise slide interpretation between sites. A quality assessment of over $10 \%$ of slides was organised at 6-month intervals by the reference pathology laboratory at Montpellier University Hospital for both sites, in addition to existing internal and external quality assurance schemes adhered to by the National Health Laboratory Service (NHLS) in SA.

The HARP end point committee, composed of five pathologists, reviewed all histological slides from women with a local diagnosis of CIN $2+$ and $\sim 10 \%$ of slides from women with normal or CIN1 histological findings; the final classification of lesions was based on a consensus of the committee.

Data analysis. Women included in this analysis comprise all of the HARP participants who were not lost to follow-up at M18 visit, including women who may have been treated for CIN2 + detected at baseline, except if this had been by hysterectomy. Proportions were compared between groups using $\chi^{2}$ or Fisher's exact test, as appropriate. Sensitivity, specificity, positive and negative predictive values (NPV) were calculated with exact binomial 95\% confidence intervals (CI), separately for each country first and then for both countries combined (sensitivity and specificity only). In addition, sensitivity and specificity analysis to detect CIN2 + was stratified 
by levels of CD4 T-cell counts ( $\leqslant 200$ cells per $\mathrm{mm}^{3}, 201-350$ cells per $\mathrm{mm}^{3},>350$ cells per $\mathrm{mm}^{3}$ ) at entry in the study and at the time of screening (M18), and by age ( $<35$ and $\geqslant 35$ years) and compared across strata using $\chi^{2}$ or Fisher's exact tests as appropriate. The comparative analysis of performance of all other methods and triage combinations for the detection of CIN2 + is not reported in this paper. Agreement between the careHPV assay and the INNO-LiPA HPV genotyping Extra assay was assessed by percentage overall agreement and prevalence-adjusted biasadjusted (PABA)-kappa coefficient. All analyses were done using the Stata version 14 software (Stata Corp, College Station, TX, USA).

\section{RESULTS}

A total of 1249 WLHA were enrolled in the study between November 2011 and October 2012, 625 in BF and 624 in SA. The analysis was based on the 1052 women (94\% overall; $\mathrm{BF}=492$; $\mathrm{SA}=560$ ) who had an adequate careHPV result at the M18 visit, which actually occurred at a median 16 months (interquartile range (IQR), 15.5-16.8; Figure 1). Among those with valid
careHPV result, $929(88 \% ; \mathrm{BF}=426, \mathrm{SA}=503)$ also had valid cytology results, and 976 returned for colposcopy $(93 \%$; $\mathrm{BF}=469$, $\mathrm{SA}=507)$. Adequate biopsies were taken from 718 (74\%) women, $265(57 \%)$ in $\mathrm{BF}$ and $453(89 \%)$ in SA. A total of 225 women $(\mathrm{BF}=179 ; \mathrm{SA}=46)$ did not require biopsy as they had no abnormal cytology, VIA/VILI or colposcopy findings and a negative careHPV test; they were classified as having 'normal/ negative' histology. Thirty three women $(\mathrm{BF}=25 ; \mathrm{SA}=8)$ did not have biopsy taken for reasons such as pregnancy, pain, cervix atrophy or stenosis. Overall, valid histology results were available for 943/1052 (90\%) women, 444 (90\%) in BF and 499 (89\%) in SA. These women had a median (IQR) CD4 + T-cell count of 495 (355-684) cells per $\mathrm{mm}^{3}, 583(412-813)$ cells per $\mathrm{mm}^{3}$ in BF and $438(331-571)$ cells per $\mathrm{mm}^{3}$ in SA. The number of women on ART was $796(75 \%), 400(81 \%)$ in BF and $396(71 \%)$ in SA, including those who were initiated onto treatment during the study period.

Overall, $45.1 \%(474 / 1052)$ of women had a positive careHPV test, $46.5 \%(229 / 492)$ in BF and $43.8 \%(245 / 560)$ in SA $(P=0.36)$. Any HPV DNA was detected in $83.4 \%$ (877/1052) cervical samples, $(80.3 \%(395 / 492)$ in BF and $86.1 \%(482 / 560)$ in SA) by the INNOLiPA genotyping Extra assay. When considering only the 14

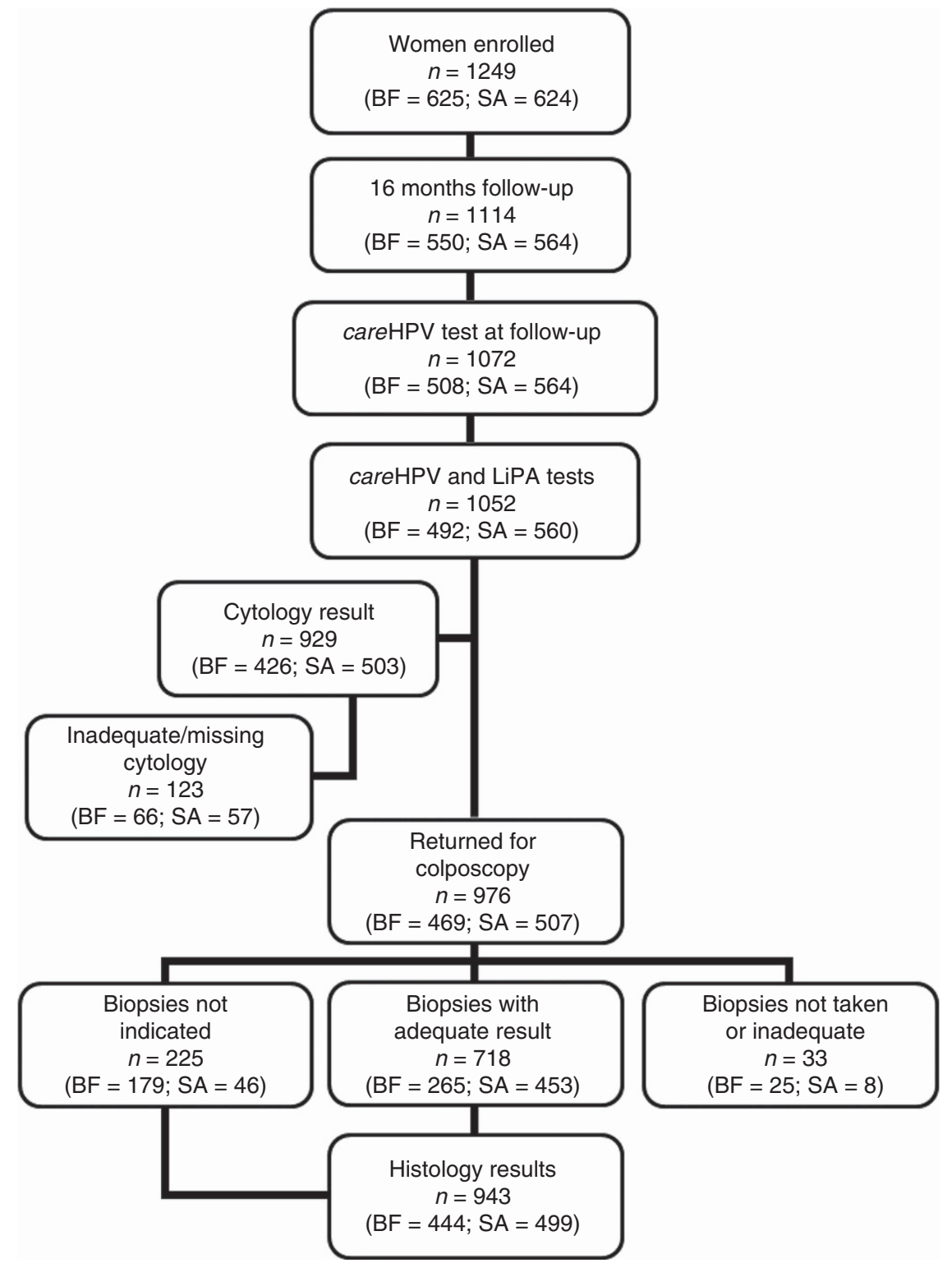

Figure 1. Study flowchart. BF = Burkina Faso; $S A=$ South Africa. 
HR-HPV types targeted by careHPV, 70.0\% (736/1052) of samples (67.9\% (334/492) in BF and 71.8\% (402/560) in SA) were positive by genotyping. The overall agreement between the two tests for HR-HPV DNA detection was $62.4 \%$, with a PABA-kappa value of 0.25 (95\% CI: 0.19-0.31) indicating a fair agreement. However, when analysis was restricted to the 60 women with CIN2 +, the agreement between tests was $93.3 \%$ with a PABA-kappa value of

Table 1. Detection rate of the 14 high-risk HPV types by the careHPV test among samples with single-type HPV infection as identified by the INNO-LiPA HPV genotyping Extra assay

\begin{tabular}{|l|r|r|r|}
\hline & \multirow{2}{*}{ INNO-LiPA } & \multicolumn{2}{c|}{ careHPV } \\
\cline { 3 - 4 } HPV types & No. & No. & $\%$ \\
\hline HPV16 & 41 & 19 & 46.3 \\
\hline HPV18 & 33 & 16 & 48.5 \\
\hline HPV31 & 49 & 22 & 44.9 \\
\hline HPV33 & 16 & 10 & 62.5 \\
\hline HPV35 & 36 & 24 & 66.7 \\
\hline HPV39 & 21 & 9 & 42.9 \\
\hline HPV45 & 20 & 16 & 80.0 \\
\hline HPV51 & 23 & 12 & 52.2 \\
\hline HPV52 & 83 & 43 & 51.8 \\
\hline HPV56 & 25 & 10 & 40.0 \\
\hline HPV58 & 22 & 19 & 86.4 \\
\hline HPV59 & 3 & 2 & 66.7 \\
\hline HPV66 & 27 & 15 & 55.6 \\
\hline HPV68 & 23 & 10 & 43.5 \\
\hline Overall & 736 & 408 & 55.4 \\
\hline Abbreviation: HPV = human papillomavirus. & & \\
\hline & & & \\
\hline
\end{tabular}

0.87 (95\% CI: 0.74-0.99) indicating an excellent agreement. Compared with the INNO-LiPA HPV genotyping Extra assay, analytical sensitivity of careHPV for detecting HR-HPV DNA was 55.4\% (95\% CI: $51.8-59.1 \%$ ). The positivity rates of the careHPV test according to the HR-HPV types detected by genotyping in single HR-HPV infection cases are presented in Table 1. The detection rates ranged from 40.0 (HPV56) to 86.4\% (HPV58).

Cytology results showed that the overall prevalence of highgrade squamous intraepithelial lesions (HSIL) was 10.5\% (98/929) with a prevalence of $2.1 \%(9 / 426)$ in BF and of $17.7 \%(89 / 503)$ in SA. As shown in Table 2, the prevalence of HR-HPV detected by careHPV or the INNO-LiPA genotyping Extra assay increased with the lesion grade $(P<0.0001)$, and the sensitivity and specificity values, combined for both countries, for the detection of HSIL were $88.8 \%(87 / 98)$ and $61.8 \%(514 / 831)$ for careHPV, and $91.8 \%$ $(90 / 98)$ and $33.7 \%(280 / 831)$, respectively, for INNO-LIPA genotyping Extra (14 types). The sensitivity of careHPV for detecting HSIL was lower in SA than in BF (87.6\% vs $100 \%)$ but specificity was higher (64.7\% vs $56.3 \%)$. Negative predictive values for HSIL were 100\% (95\% CI: $98.4-100.0)$ and $96.1 \%$ (95\% CI: 93.1-98.0) in BF and SA, respectively.

A total of $60(6.4 \%)$ women had high-grade (CIN2+) histological lesions, $9(2.0 \%)$ in $\mathrm{BF}$ and $51(10.2 \%)$ in SA $(P<0.0001)$. Overall, the careHPV test was positive in $56 / 60$ (93.3\%) women with CIN2+, $9(100 \%)$ in BF and $47(92.2 \%)$ in SA, respectively $(P>0.5)$. The performance characteristics of the careHPV and INNO-LiPA genotyping Extra assays for the diagnosis of CIN2 + in each country and overall are presented in Table 3. The NPVs of both tests were very similar (98.6-100\%), and when combining the results for the two countries, the careHPV test was slightly less sensitive (93.3\% vs 96.7\%) but more specific $(57.9 \%$ vs $32.9 \%)$ than the INNO-LiPA HPV genotyping Extra assay. Stratification of results by CD $4+$ T-cell count showed that there was no significant difference in sensitivities by CD $4+$ T-cell count levels at study entry or contemporary to CIN biopsy at

Table 2. Prevalence of HR-HPV detection by careHPV and INNO-LiPA genotyping Extra assay according to cytological results

\begin{tabular}{|c|c|c|c|c|c|c|}
\hline \multirow[b]{2}{*}{ Cytology } & \multicolumn{3}{|c|}{ Burkina Faso $(n=426)$} & \multicolumn{3}{|c|}{ South Africa $(n=503)$} \\
\hline & No. & careHPV-positive (\%) & INNO-LiPA-positive (\%) & No. & careHPV-positive (\%) & INNO-LiPA-positive (\%) \\
\hline No anomalies & 368 & $148(40.2)$ & $240(65.2)$ & 14 & $4(28.6)$ & $6(42.9)$ \\
\hline Atypical cells ${ }^{a}$ & 16 & $9(56.2)$ & $12(75.0)$ & 84 & 28 (33.3) & $58(69.0)$ \\
\hline LSIL & 33 & $25(75.8)$ & $28(84.8)$ & 316 & $114(36.1)$ & $215(68.0)$ \\
\hline $\mathrm{HSIL}+$ & 9 & $9(100)$ & $9(100)$ & 89 & $78(87.6)$ & $81(91.0)$ \\
\hline$P^{b}$ & & $<0.0001$ & 0.0016 & & $<0.0001$ & 0.0011 \\
\hline \multicolumn{7}{|c|}{$\begin{array}{l}\text { Abbreviations: } B F=\text { Burkina Faso; } \mathrm{HPV}=\text { human papillomavirus; } \mathrm{HSIL}=\text { high-grade squamous intraepithelial lesions; } \mathrm{LSIL}=\text { low-grade squamous intraepithelial lesions; } \mathrm{SA}=\text { South Africa. } \\
\text { a Atypical squamous cells of undetermined significance (ASC-US), } n=13(\mathrm{BF}) \text { and } n=52(\mathrm{SA}) \text {; Atypical squamous cells, cannot exclude HSIL (ASC-H), } n=2(\mathrm{BF}) \text { and } n=32(\mathrm{SA}) ; A \text { typical } \\
\text { glandular cells (AGC), } n=1 \text { (BF). } \\
\text { best for trend. }\end{array}$} \\
\hline
\end{tabular}

Table 3. Performance of the careHPV and INNO-LiPA assays for the diagnosis of CIN2 + lesions $(n=943)$

\begin{tabular}{|c|c|c|c|c|c|c|}
\hline & \multicolumn{2}{|c|}{ Burkina Faso $(\mathrm{CIN} 2+, n=9)$} & \multicolumn{2}{|c|}{ South Africa (CIN2,$+ n=51)$} & \multicolumn{2}{|c|}{ Overall $(\mathrm{CIN} 2+, n=60)$} \\
\hline & careHPV & INNO-LiPA & careHPV & INNO-LiPA & careHPV & INNO-LiPA \\
\hline \multicolumn{7}{|l|}{ Performance indicators } \\
\hline No. of positive tests & 206 & 300 & 222 & 358 & 428 & 658 \\
\hline No. of CIN2 + positive by test & 9 & 8 & 47 & 50 & 56 & 58 \\
\hline Sensitivity \% & 100 (66.4-100) & 88.9 (51.7-99.7) & $92.2(81.1-97.8)$ & $98.0(89.6-99.6)$ & $93.3(83.8-98.2)$ & 96.7 (88.5-99.6) \\
\hline Specificity \% & $54.7(49.9-59.5)$ & $32.9(28.5-37.5)$ & $60.9(56.3-65.5)$ & $31.3(27.0-35.8)$ & $57.9(54.5-61.2)$ & $32.0(29.0-35.2)$ \\
\hline PPV \% & $4.4(2.0-8.1)$ & $2.7(1.2-5.2)$ & $21.2(16.0-27.1)$ & $14.0(10.6-18.0)$ & $-{ }^{a}$ & - \\
\hline NPV \% & $100(98.5-100)$ & $99.3(96.2-100)$ & $98.6(96.3-99.6)$ & $99.3(96.1-100)$ & - & - \\
\hline
\end{tabular}


M18. However, specificities decreased with decreasing CD4+ T-cell counts both at study enrolment $(P=0.01)$ and at M18 $(P=0.0008)$, which corresponded to increasing HR-HPV prevalences (Table 4). Participants on ART at the time of enrolment had lower HR-HPV prevalence at M18. care HPV was slightly more specific in these women $(P=0.05)$. There were no significant difference by age (Table 4$)$.

\section{DISCUSSION}

Considering the high prevalence of HIV infection in sub-Saharan Africa and the fact that WLHA have an increased risk of development of cervical precancerous and cancerous lesions, it is important to target this population in cervical cancer prevention programs. It is also important to verify that the performance of screening tests is not modified by HIV serostatus. Several studies conducted in China (Qiao et al, 2008), India (Labani et al, 2014), Thailand (Trope et al, 2013), Brazil (Lorenzi et al, 2013) and Nigeria (Gage et al, 2012) on clinicianor patient-collected cervical samples have shown that cervical screening based on careHPV testing was a feasible and performant strategy in low-resource settings. However, none of these studies had been conducted among WLHA. Thus, the possible impact of HIV infection on the performance of careHPV for cervical screening deserved further investigation.

As expected, the positivity rate of careHPV increased with the grade of cytological lesions, ranging from 87.6 (SA) to $100 \%(\mathrm{BF})$ in women with HSIL. The end point in this study was CIN2 + and our results indicate that careHPV detected CIN2 + with a high sensitivity $(93.3 \%)$ and a high NPV $(\geqslant 99 \%)$ in this population of WLHA. The sensitivity of careHPV for detecting CIN2 + observed in the present study was very similar to that (94.3\%) recently reported in the only other study among WLHA, although specificity was not reported in that Ugandan study (Bansil et al, 2015). The overall specificity of careHPV was relatively low (58\%) and varied by CD 4 T-cell strata (between 43 and 62\%). A similar finding, albeit with even lower specificity, was reported in a study of a similar group of WLHA in Johannesburg tested with HC2 (Firnhaber et al, 2013). The high prevalence of HR-HPV in this highly exposed population, which increases by level of immune suppression, may lead to low test specificity, as not all HPV infections will progress to CIN2 + lesions, or only much later.
Comparison of the careHPV test with the INNO-LiPA HPV genotyping Extra assay showed that careHPV is less sensitive than the genotyping assay for the detection of HR-HPV DNA, and that the rate of detection varies according to the different HR-HPV types. However, despite the higher analytical sensitivity of the INNO-LiPA assay for HR-HPV DNA detection, the clinical sensitivity of the two tests for the diagnosis of CIN2 + was very comparable and the careHPV test had superior specificity. We may infer that the observed differences in analytical sensitivity of careHPV may not affect its usefulness as a screening test. We have previously reported that the $\mathrm{HC} 2$ assay, from which the careHPV test is derived, had an overall sensitivity and specificity of $88.8 \%$ and $55.2 \%$, respectively, to detect CIN2 + lesions in this study population at their first round of screening (Ngou et al, 2015), which would make that HPV assay suitable for screening. The overall sensitivity and specificity of 93.3 and $57.9 \%$ observed in the present study indicate that careHPV would perform equally well for cervical cancer screening of WLHA in subSaharan Africa. Given the relative low specificity of HPV testing, a triage test such as cytology might be required to determine which women should be referred to colposcopy. The cost-effectiveness of this approach should be evaluated.

The high prevalence of HR-HPV infection and CIN2+ observed in this study are in agreement with data obtained among similar populations in sub-Saharan Africa (Hawes et al, 2003; Didelot-Rousseau et al, 2006; Singh et al, 2009; Firnhaber et al, 2010; De Vuyst et al, 2012). Interestingly, while HR-HPV prevalence as determined by careHPV was not significantly different between BF and SA, prevalence of cytological and histological high-grade lesions were markedly higher in SA compared with BF. A similarly high prevalence of lesions among HIV-1-infected women in SA has been already reported (Firnhaber et al, 2013) and factors that may explain the differences observed between these two countries will be further investigated. At least in the HARP study, we can rule out issues of histological misclassification as final histological diagnosis of CIN2 + lesions was established by a consensus Expert Committee reviewing slides from both countries simultaneously.

In conclusion, our results indicate that the careHPV test would be a reliable tool for cervical cancer screening in WLHA. Such a cost-affordable test should be considered for implementation in cervical cancer prevention programs in sub-Saharan Africa targeting women living with HIV/AIDS.

Table 4. Effect of baseline or contemporary CD4 count, age and antiretroviral therapy (ART) on the performance of careHPV

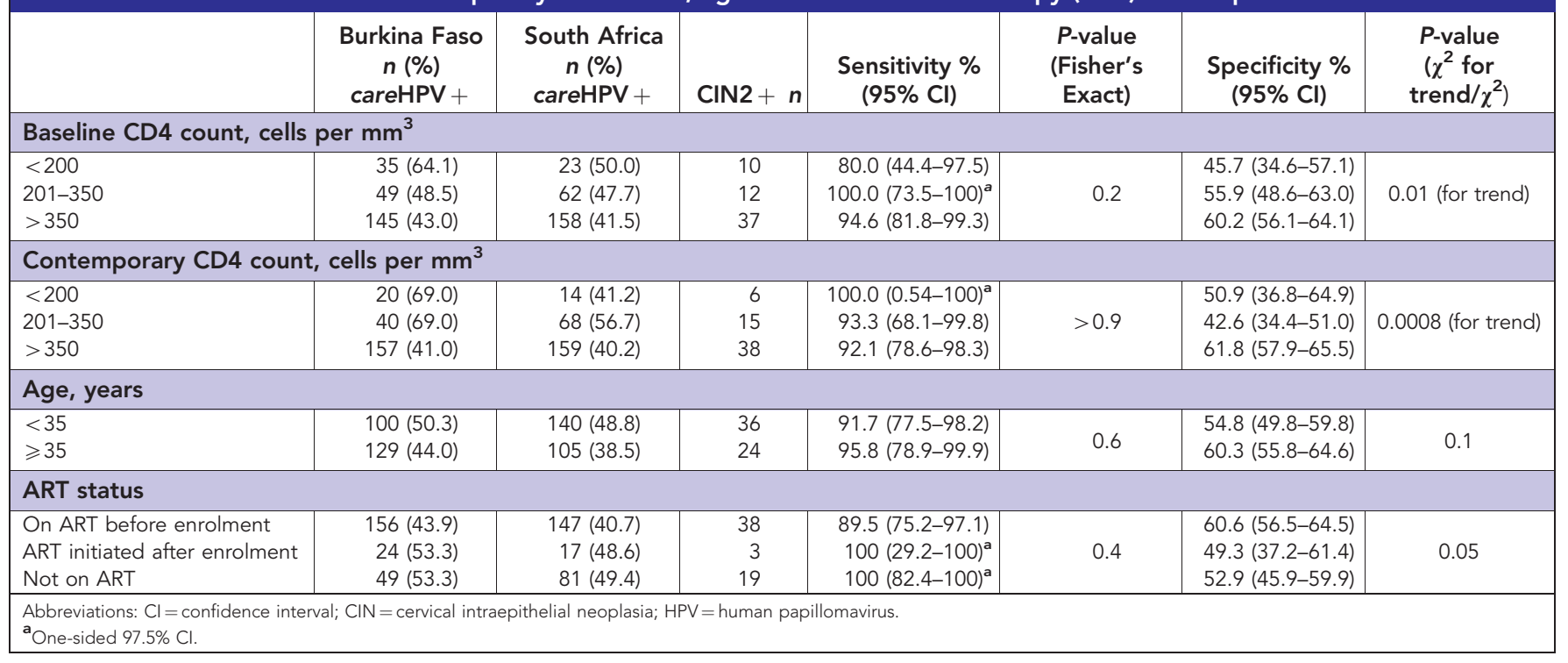




\section{ACKNOWLEDGEMENTS}

Other contributing members of the HARP study group included: E Cutler, D Lewis, V Meseko, B Muzah and A Puren (Johannesburg, South Africa); J Drabo, J Simporé, A Yonli and S Zan (Ouagadougou, Burkina Faso); and A Devine, LJ Gibson, R Legood, and HA Weiss (London, UK); and N Nagot (Montpellier, France). We wish to thank the members of the HARP International Scientific Advisory Group (ISAG): C Lacey (University of York, UK), M Chirenje (University of Harare, Zimbabwe),Y Qiao (Chinese Academy of Medical Sciences and Peking Union Medical College, Beijing, China) and $\mathrm{S}$ de Sanjosé (Institut Catala d'Oncologia, Barcelona, Spain). We also thank $\mathrm{H}$ Perrochia (Montpellier, France) for her participation in the histological classification end point committee. careHPV test kits and testing systems were obtained through a Qiagen Corporation donation programme. The research leading to these results has received funding from the European Commission (EC) 7th Framework Programme under grant agreement No. HEALTH-2010-F2-265396.

\section{CONFLICT OF INTEREST}

The authors declare no conflict of interest.

\section{REFERENCES}

Bansil P, Lim J, Byamugisha J, Kumakech E, Nakisige C, Jeronimo JA (2015) Performance of cervical cancer techniques in HIV-infected women in Uganda. J Low Genit Tract Dis 19: 215-219.

Chen W, Jeronimo J, Zhao FH, Qiao YL, Valdez M, Zhang X, Kang LN, Bansil P, Paul P, Bai P, Peck R, Li J, Chen F, Stoler MH, Castle PE (2014) The concordance of HPV DNA detection by Hybrid Capture 2 and careHPV on clinician- and self-collected specimens. J Clin Virol 61: 553-557.

Cuzick J, Arbyn M, Sankaranarayanan R, Tsu V, Ronco G, Mayrand MH, Dillner J, Meijer CJ (2008) Overview of human papillomavirus-based and other novel options for cervical cancer screening in developed and developing countries. Vaccine 26(Suppl 10): K29-K41.

Denny L, Anorlu R (2012) Cervical cancer in Africa. Cancer Epidemiol Biomarkers Prev 21: 1434-1438.

De Vuyst H, Lillo F, Broutet N, Smith JS (2008) HIV, human papillomavirus, and cervical neoplasia and cancer in the era of highly active antiretroviral therapy. Eur J Cancer Prev 17: 545-554.

De Vuyst H, Mugo NR, Chung MH, McKenzie KP, Nyongesa-Malava E, Tenet V, Njoroge JW, Sakr SR, Meijer CM, Snijders PJ, Rana FS, Franceschi S (2012) Prevalence and determinants of human papillomavirus infection and cervical lesions in HIV-positive women in Kenya. Br J Cancer 107: 1624-1630.

Didelot-Rousseau MN, Nagot N, Costes-Martineau V, Vallès X, Ouedraogo A, Konate I, Weiss HA, Van de Perre P, Mayaud P, Segondy M (2006) Human papillomavirus genotype distribution and cervical squamous intraepithelial lesions among high-risk women with and without HIV-1 infection in Burkina Faso. Br J Cancer 95: 355-362.

Ferlay J, Soerjomataram I, Dikshit R, Eser S, Mathers C, Rebelo M, Parkin DM, Forman D, Bray F (2015) Cancer incidence and mortality worldwide: sources, methods and major patterns in GLOBOCAN 2012. Int J Cancer 136: E359-E386.

Firnhaber C, Mayisela N, Mao L, Williams S, Swarts A, Faesen M, Levin S, Michelow P, Omar T, Hudgens MG, Williamson AL, Allan B, Lewis DA,
Smith JS (2013) Validation of cervical cancer screening methods in HIV positive women from Johannesburg South Africa. PLoS One 8: e53494.

Firnhaber C, Van Le H, Pettifor A, Schulze D, Michelow P, Sanne IM, Lewis DA, Williamson AL, Allan B, Williams S, Rinas A, Levin S, Smith JS (2010) Association between cervical dysplasia and human papillomavirus in HIV seropositive women from Johannesburg South Africa. Cancer Causes Control 21: 433-443.

Gage JC, Ajenifuja KO, Wentzensen N, Adepiti AC, Stoler M, Eder PS, Bell L, Shrestha N, Eklund C, Reilly M, Hutchinson M, Wacholder S, Castle PE, Burk RD, Schiffman M (2012) Effectiveness of a simple rapid human papillomavirus DNA test in rural Nigeria. Int J Cancer 131: 2903-2909.

Hawes SE, Critchlow CW, Faye Niang MA, Diouf MB, Diop A, Touré P, Aziz Kasse A, Dembele B, Salif Sow P, Coll-Seck AM, Kuypers JM, Kiviat NB (2003) Increased risk of high-grade cervical squamous intraepithelial lesions and invasive cancer among African women with human immunodeficiency virus type 1 and 2 infections. J Infect Dis $\mathbf{1 8 8}$ : $555-563$.

Labani S, Asthana S, Sodhani P, Gupta S, Bhambhani S, Pooja B, Lim J, Jeronimo J (2014) CareHPV cervical cancer screening demonstration in a rural population of north India. Eur J Obstet Gynecol Reprod Biol 176: 75-79.

Lin CQ, Chen F, Liu B, Zhang YZ, Cui XL, Li AM, Zhang WH, Chen W, Chang I, Sivasubramaniam P, Zhu J, Qiao YL (2014) A parallel study of careHPV and Hybrid Capture2 human papillomavirus DNA testing for cervical cancer screening in rural China. J Virol Methods 202: 73-78.

Lorenzi AT, Fregnani JH, Possati-Resende JC, Neto CS, Villa LL, LongattoFilho A (2013) Self-collection for high-risk HPV detection in Brazilian women using the careHPV ${ }^{\mathrm{TM}}$ test. Gynecol Oncol 131: 131-134.

Ngou J, Gilham C, Omar T, Goumbri-Lompo O, Doutre S, Michelow P, Kelly H, Didelot MN, Chikandiwa A, Sawadogo B, Delany-Moretlwe S, Meda N, Costes V, Mayaud P, Segondy M (2015) Comparison of analytical and clinical performances of the Digene HC2 HPV DNA assay and the INNO-LiPA HPV genotyping assay for detecting high-risk HPV infection and cervical neoplasia among HIV-positive African women J Acquir Immune Defic Syndr 68: 162-168.

Ngou J, Magooa MP, Gilham C, Djigma F, Didelot MN, Kelly H, Yonli A, Sawadogo B, Lewis DA, Delany-Moretlwe S, Mayaud P, Segondy M (2013) Comparison of careHPV and hybrid capture 2 assays for detection of high-risk human Papillomavirus DNA in cervical samples from HIV-1infected African women. J Clin Microbiol 51: 4240-4242.

Qiao YL, Sellors JW, Eder PS, Bao YP, Lim JM, Zhao FH, Weigl B, Zhang WH, Peck RB, Li L, Chen F, Pan QJ, Lorincz AT (2008) A new HPV-DNA test for cervical-cancer screening in developing regions: a cross-sectional study of clinical accuracy in rural China. Lancet Oncol 9: 929-936.

Ronco G, Dillner J, Elfström KM, Tunesi S, Snijders PJ, Arbyn M, Kitchener H, Segnan N, Gilham C, Giorgi-Rossi P, Berkhof J, Peto J, Meijer CJ (2014) International HPV screening working group. Efficacy of HPV-based screening for prevention of invasive cervical cancer: follow-up of four European randomised controlled trials. Lancet 383: 524-532.

Singh DK, Anastos K, Hoover DR, Burk RD, Shi Q, Ngendahayo L, Mutimura E, Cajigas A, Bijirimani V, Cai X, Rwamwejo J, Vuolo M, Cohen M, Castle PE (2009) Human papillomavirus infection and cervical cytology in HIV-infected and HIV-uninfected Rwandan women. J Infect Dis 199: 1851-1861.

Smith JH (2002) Bethesda 2001. Cytopathology 13: 4-10.

Trope LA, Chumworathayi B, Blumenthal PD (2013) Feasibility of community-based careHPV for cervical cancer prevention in rural Thailand. J Low Genit Tract Dis 17: 315-319.

This work is published under the standard license to publish agreement. After 12 months the work will become freely available and the license terms will switch to a Creative Commons AttributionNonCommercial-Share Alike 4.0 Unported License. 